Bond University

Research Repository

\title{
A new ecosystem for evidence synthesis
}

Nakagawa, Shinichi; Dunn, Adam G; Lagisz, Malgorzata; Bannach-Brown, Alexandra; Grames, Eliza M; Sánchez-Tójar, Alfredo; O'Dea, Rose E; Noble, Daniel W A; Westgate, Martin J; Arnold, Pieter A; Barrow, Stuart; Cooper, Eve; Foo, Yong Zhi; Geange, Sonya R; Hennessy, Emily; Mapanga, Witness; Mengersen, Kerrie; Munera, Claudia; Page, Matthew J; Welch, Vivian; Evidence Synthesis Hackathon 2019 Participants; Haddaway, Neal R Published in:

Nature Ecology and Evolution

DOI:

$10.1038 / s 41559-020-1153-2$

Licence:

Other

Link to output in Bond University research repository.

Recommended citation(APA):

Nakagawa, S., Dunn, A. G., Lagisz, M., Bannach-Brown, A., Grames, E. M., Sánchez-Tójar, A., O'Dea, R. E., Noble, D. W. A., Westgate, M. J., Arnold, P. A., Barrow, S., Cooper, E., Foo, Y. Z., Geange, S. R., Hennessy, E., Mapanga, W., Mengersen, K., Munera, C., Page, M. J., ... Haddaway, N. R. (2020). A new ecosystem for evidence synthesis. Nature Ecology and Evolution, 4, 498-501. https://doi.org/10.1038/s41559-020-1153-2

\section{General rights}

Copyright and moral rights for the publications made accessible in the public portal are retained by the authors and/or other copyright owners and it is a condition of accessing publications that users recognise and abide by the legal requirements associated with these rights.

For more information, or if you believe that this document breaches copyright, please contact the Bond University research repository coordinator. 


\section{Title: A New Ecosystem for Evidence Synthesis}

Shinichi Nakagawa* ${ }^{*}$, Adam G. Dunn ${ }^{2}$, Malgorzata Lagisz ${ }^{1}$, Alexandra Bannach-Brown ${ }^{3}$, Eliza M. Grames ${ }^{4}$, Alfredo Sánchez-Tójar ${ }^{5}$, Rose O'Dea ${ }^{1}$, Daniel W. A. Noble ${ }^{6}$, Martin J. Westgate ${ }^{7}$, Pieter A. Arnold ${ }^{6}$, Stuart Barrow ${ }^{8}$, Alison Bethel ${ }^{9}$, Eve Cooper ${ }^{6}$, Yong Zhi Foo ${ }^{1}$, Sonya Geange ${ }^{6}$, Emily Hennessy ${ }^{10}$, Witness Mapanga ${ }^{11}$, Kerrie Mengersen ${ }^{12}$, Claudia Munera ${ }^{7}$, Matthew J. Page ${ }^{13}$, Vivian Welch ${ }^{14}$, Evidence Synthesis Hackathon 2019 Participants $^{\star *}$ \& Neal R. Haddaway ${ }^{15,16}$

* corresponding author: s.nakagawa@unsw.edu.au (ORCID: 0000-0002-7765-5182)

** the list of contributors appears at the end of the article

${ }^{1}$ Evolution \& Ecology Research Centre, School of Biological, Earth and Environmental Sciences, University of New South Wales, Sydney, NSW, Australia

${ }^{2}$ Centre for Health Informatics, Macquarie University, Sydney, NSW, Australia

${ }^{3}$ Institute for Evidence-Based Healthcare, Bond University, Gold Coast, QLD, Australia

${ }^{4}$ Department of Ecology \& Evolutionary Biology, University of Connecticut, Storrs, Connecticut, USA

${ }^{5}$ Department of Evolutionary Biology, Bielefeld University, Germany

${ }^{6}$ Division of Ecology and Evolution, Research School of Biology, The Australian National University, Canberra, ACT, Australia

${ }^{7}$ Fenner School of Environment \& Society, The Australian National University, Canberra, ACT, Australia

${ }^{8}$ Australian Academy of Science, Canberra, ACT, Australia

${ }^{9}$ College of Medicine and Health, University of Exeter, UK

${ }^{10}$ Department of Psychological Sciences, Institute for Collaboration on Health, Intervention, and Policy, University of Connecticut, Storrs, CT, USA

${ }^{11}$ Centre for Health Policy, School of Public Health, University of Witwatersrand, Johannesburg, South Africa

${ }^{12}$ School of Mathematical Sciences, Queensland University of Technology, Brisbane, QLD, Australia

${ }^{13}$ School of Public Health and Preventive Medicine, Monash University, Melbourne, VIC, Australia

${ }^{14}$ Campbell Collaboration

${ }^{15}$ Stockholm Environment Institute, Linnégatan 87D, Stockholm, Sweden

${ }^{16}$ Africa Centre for Evidence, University of Johannesburg, Johannesburg, South Africa 


\section{Synthesizing evidence is an essential part of scientific progress, but it is often done in a slow and uncoordinated manner, sometimes producing misleading conclusions. Here, we propose an idea of 'open synthesis community' to resolve this pressing issue.}

In the acclaimed BBC documentary Life of Birds (1998), David Attenborough narrated male house sparrows displaying a black patch of feathers on their chest or bib as a 'badge of status' to signal their fighting ability. Ten years later, a synthesis of published studies confirmed that the bigger the patch, the higher the social status. However, another ten years later an updated synthesis included unpublished studies, and questioned whether the bib signals their status at all'.

This sparrow example may seem benign but consider the following example. An early synthesis of clinical trial evidence led governments to spend billions of dollars stockpiling the anti-flu drug Tamiflu ${ }^{2}$. It took another ten years to access the unpublished data that showed Tamiflu was probably not as effective, or as safe, as was first believed ${ }^{2}$. There are an increasing number of examples of evidence weakening or disappearing over time, not only in the field of ecology and evolution, but also in other fields ${ }^{3}$. This phenomenon is called a 'decline effect'. Such a phenomenon suggests that we should reconsider the way we synthesize evidence.

Evidence synthesis, such as a systematic review, aims to integrate all available research articles to summarise research knowledge and test or generate new hypotheses. The process of evidence synthesis is often slow and laborious ${ }^{4}$, and biases are introduced when primary research remains unreported or otherwise inaccessible for synthesis. Usually, a large group of primary researchers (empiricists, hereafter) collect data and they may or may not publish their work. Then, a smaller group of synthesists try to find and compile all relevant studies, often without empiricists being aware. Both parties spend many years worth of work, independently, before reliable evidence and consensus emerge on a topic.

To change this inefficient process we propose to overhaul the current ecosystem of how evidence synthesis is created, forging a community between empiricists and synthesists. First, we briefly discuss how current inefficiencies can affect society. Then, we illustrate the benefits of our proposal by discussing our future vision and what actions academic societies, 
universities, and funding agencies can take. Although we preferentially draw examples from the field of ecology and evolution, our future vision is broadly applicable across fields.

\section{Where the status quo may be taking us}

It has been nearly impossible to keep up with the deluge of information made available to support not only our academic tasks but also our daily decisions. To manage the day-to-day information overload, we rely on algorithms to decide for us what information we want to see. Google uses our search history to decide what is relevant, Reddit and other forum-like websites use voting mechanisms to draw on the wisdom of the crowd, and Amazon predicts what we will want by looking at what people like us have already selected.

The algorithms we rely on to filter and select the day-to-day information have developed rapidly and are seamlessly integrated into our lives. The methods we use to synthesize research evidence, however, are much more complex, and therefore harder to automate ${ }^{5}$. Rather than simply identifying the most relevant information, evidence syntheses need to find all relevant information, make sense of it, identify potential biases, and summarise it for consumption. To meet the challenge of streamlining evidence synthesis, we clearly need tools to do it as fast as possible, but we also need to make sure we are synthesising the right evidence at the right time in the right way to avoid waste and, more importantly, to avoid implementing 'wrong' practice or science.

There has been a recent suggestion that, in biomedical research, many systematic reviews and meta-analyses are redundant, unnecessary, or misleading ${ }^{6}$. While this has been studied less outside of medical research, we expect that it is already happening in the field of ecology and evolution ${ }^{7}$. The inability to recognise gaps where evidence is needed and to avoid repeating systematic reviews that have already been written is likely due to a lack of communication between empiricists and synthesists as well as competition and a lack of communication between synthesists. This competition and lack of integration are expected to lead to the poor translation of research findings into policy outcomes. Then, we cannot completely blame policymakers for not adopting more evidence-based approaches in their decision making process because they must often rely on dubious, premature, scattered, and outdated sources of evidence. 


\section{Our future vision: an open synthesis community}

In the future, we envision a new research ecosystem where every empiricist is contributing to evidence synthesis by being part of a community. This community comprises a group of researchers who engage in research synthesis on their study topics with support personnel, such as librarians, statisticians, and computer programmers. Where researchers undertaking primary research are part of such a community, synthesis is recognised as the end goal, as researchers design, undertake, and report their work (cf. ${ }^{8}$ ).

Consider a graduate student researching the effect of deforestation on insect communities. As part of their research, they carry out experiments to collect data. But in a new research ecosystem the student is also embedded in a synthesis community studying deforestation and related issues. Mentors from the community guide the student on how their new research fits within the current body of work and help ensure that their primary research is synthesizable ${ }^{9}$. With community guidance, they upload information about their research designs, results, and other research materials to a database regardless of the statistical significance of their results. In this way, the student takes responsibility for ensuring their data can be readily discovered and integrated into relevant ongoing research syntheses and their contributions are recognised through authorship or contributorship on the synthesis articles that include their data (Fig. 1) ${ }^{10}$. To ensure quality and fairness, standards and checking procedures are in place to maintain the quality and objectivity of data entries to manage conflicts of interests when new primary researchers become involved in the synthesis community.

In this future scenario, evidence syntheses are updated continuously. In biomedical science, some groups have already initiated 'living' systematic reviews, where a synthesis team monitors new trial results as they are reported to rapidly incorporate new evidence in already published reviews ${ }^{11}$. Our living synthesis differs from this model because it directly embeds the whole research community into evidence synthesis in an open and transparent way.

Evidence synthesis communities will be supported with tools to facilitate the work of the community, including data visualization, and the digestion and interpretation of synthesis outcomes $^{12}$. Scientists and the public alike will be able to access and assess the most current evidence within any topic. A web-based synthesis platform will provide a way to connect researchers and interested public members, improving stakeholder engagement ${ }^{12-14}$. 


\section{Benefits of open synthesis communities}

A key advantage of this vision is the capacity to minimise research waste caused by unpublished data and redundancy ${ }^{15}$. When all researchers are part of synthesis projects, both disappointing and exciting results will be published. Redundancy and duplications in evidence synthesis will be reduced because the community will be aware of ongoing and planned synthesis work, and can work together through a fluid model of contributorship ${ }^{10}$. This model incentivises both empiricists and synthesists. Primary research data is less likely to be wasted because primary researchers will be more aware of the need to make their data FAIR (findable, accessible, interoperable and reusable ${ }^{16}$, ensuring their research is synthesizable and impactful.

A second key advantage is that open synthesis communities will be able to monitor gaps and biases in primary research and synthesis tasks. This will support improved prioritization of future projects, including needed replications, reducing research waste. By breaking down the divide between synthesists and empiricists, open synthesis communities can bring empiricists further along the journey from data collection, through synthesis, to the communication of their research to stakeholders. Rather than being 'research parasites'17, synthesists instead support empiricists through evidence synthesis processes and both groups respect and learn from each other through better communication and involvement in each others' processes. Taken together, we will start to see, in advance, how individual studies are likely to fit into the larger scientific jigsaw puzzle, and be able to better measure impact on science and society.

As this proposed community is open to all researchers, it will increase diversity and reduce inequality in the current scientific community. For example, women in science are less likely to be included in certain scientific networks, or receive similar recognition for their work ${ }^{18}$. By removing this kind of implicit gatekeeping that has existed in science, all members of the community will be included in this open synthesis community, as they start their own research, acting as a prevention for the leaky pipeline ${ }^{19}$. Further, the open synthesis community welcomes all scientists from any laboratories regardless of resources and locations, realizing that diversity promotes better science ${ }^{20}$. 


\section{How do we get there?}

We can create and maintain evidence synthesis communities through improvements in education, communication, practice and methodology, changing the status quo. Our journey to an open synthesis community will require better infrastructure and technology to promote openness and interconnectedness between various stakeholders to facilitate synthesis.

We believe scientific societies or academic institutions can provide such infrastructure by starting to organise synthesis topics and groups. They understand the needs of their scientific community assuming that they listen to their community needs. For example, an academic society can form a special committee, identifying urgent topics or topics of greatest interest to the community and then, help forming special interest groups to start initiatives. Or academic societies, with their journals (e.g. British Ecological Society and the Society for the Study of Evolution), could arrange special issues, associated with special interest groups.

Also, existing organisations overseeing evidence synthesis, such as the Collaboration for Environmental Evidence, Cochrane, and Campbell Collaboration, can take initiatives towards these goals. Funding agencies could kickstart this type of synthesis activity by forming a national centre or a centre of excellence like NCEAS (National Center for Ecological Analysis and Synthesis) and NESCent (National Evolutionary Synthesis Center). Both centers have been influential, but their model is to form exclusive expert groups rather than inclusive synthesis communities like envisioned here. These agencies could also create new funding pools that promote relevant workshops to initiate an open synthesis community.

We consider our Evidence Synthesis Hackathon events a great starting point for forming such a community. Our hackathon, which is a form of workshop, includes a diverse group of interdisciplinary scientists (medical, social, environmental, and computer scientists, biologists, librarians, and statisticians) from all around the world. At our events, we discuss and code together to advance technology to make evidence synthesis easier, less costly and timeconsuming, and more transparent and reproducible. While this community was first formed less than 3 years ago, we have already produced free-to-use synthesis tools to this end (https://www.eshackathon.org/). Though it is still relatively small, it is an initiative that is wellaligned with a much larger movement addressing the reproducibility crisis $^{13}$. 


\section{Towards an evidence revolution}

Glenn Begley was among the first people to warn the scientific community of its reproducibility crisis $^{21}$, which he optimistically refers to as an 'innovation opportunity'. Since then, researchers have taken up this opportunity and have demonstrated that they are willing to engage with open science practices, even though it may mean more work. For example, in the field of ecology and evolution, public data archiving has become mandatory ${ }^{22}$, and it is now possible to conduct a meta-analysis using archived datasets rather than summary statistics ${ }^{23}$. Now psychologists are following suit ${ }^{24}$. Simine Vazire calls such a positive change the 'credibility revolution'25. Combining this revolution with unprecedented growth in primary research, it seems just the right time to revolutionize the way we do evidence synthesis.

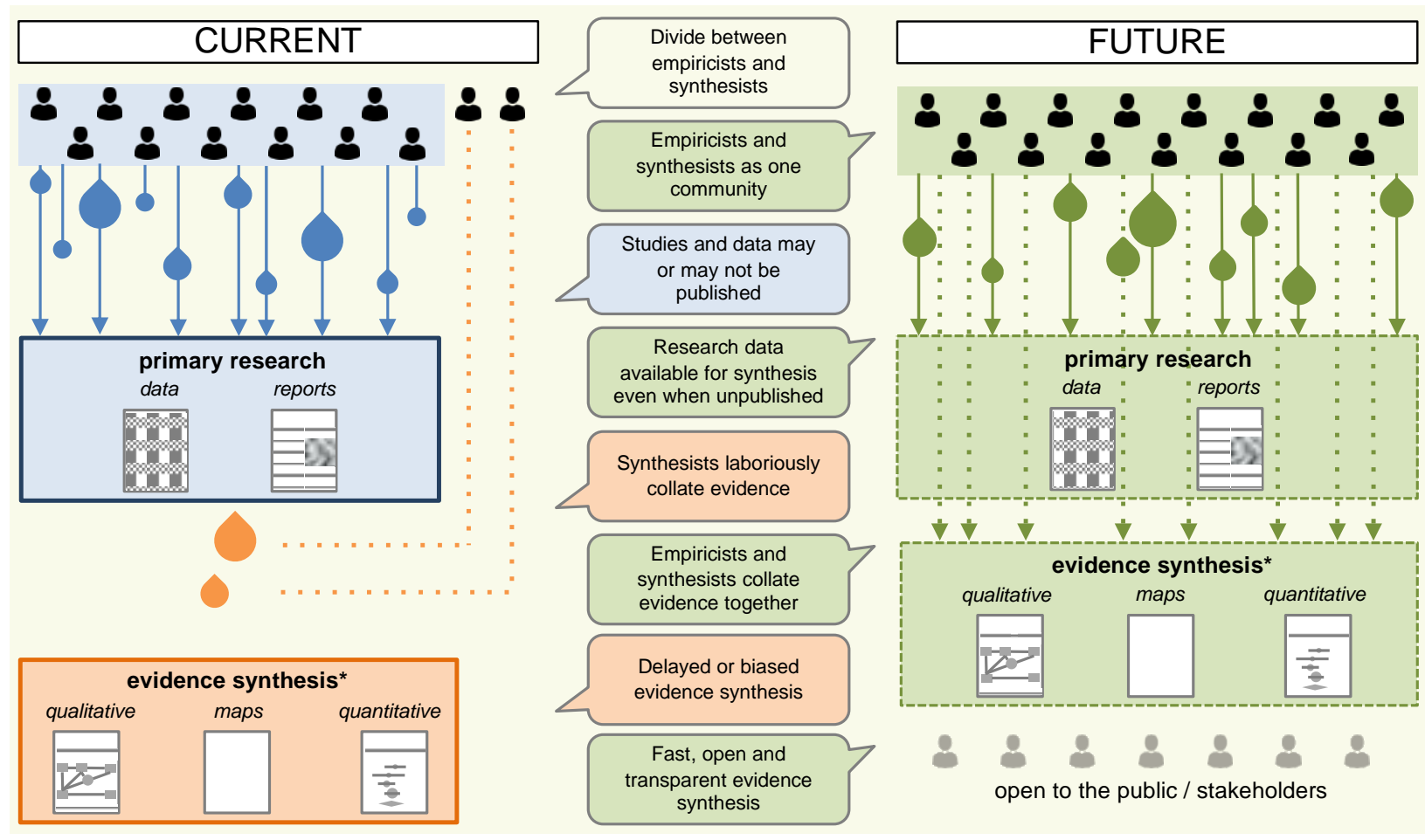

\section{Figure Legends}

Fig. 1| Open Synthesis Community. Bridging the divide between empiricists and synthesists will lead to less research waste, more collaboration, faster research progress, and better engagement. Notably, in the current ecosystem, the process of research synthesis and resulting synthesis are mostly inaccessible to the public or stakeholders, whereas the proposed future 
ecosystem includes opening up both the process and publications. *Evidence synthesis includes qualitative systematic reviews, quantitative systematic reviews (including metaanalysis), systematic maps (which catalogue research on broader topics), and other types of reviews.

\section{REFERENCES}

1. Sánchez-Tójar, A. et al. Meta-analysis challenges a textbook example of status signalling and demonstrates publication bias. eLife 7, e37385 (2018).

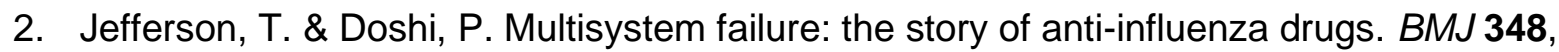
g2263 (2014).

3. Koricheva, J. \& Kulinskaya, E. Temporal Instability of Evidence Base: A Threat to Policy Making? Trends Ecol. Evol. (2019) doi:10.1016/j.tree.2019.05.006.

4. Borah, R., Brown, A. W., Capers, P. L. \& Kaiser, K. A. Analysis of the time and workers needed to conduct systematic reviews of medical interventions using data from the PROSPERO registry. BMJ Open 7, e012545 (2017).

5. Brassey, J. et al. Developing a fully automated evidence synthesis tool for identifying, assessing and collating the evidence. BMJ Evid.-Based Med. bmjebm-2018-111126 (2019) doi:10.1136/bmjebm-2018-111126.

6. Ioannidis, J. P. A. The Mass Production of Redundant, Misleading, and Conflicted Systematic Reviews and Meta-analyses. Milbank Q. 94, 485-514 (2016).

7. Gurevitch, J., Koricheva, J., Nakagawa, S. \& Stewart, G. Meta-analysis and the science of research synthesis. Nature 555, 175-182 (2018).

8. Seidler, A. L. et al. A guide to prospective meta-analysis. BMJ 367, (2019).

9. Gerstner, K. et al. Will your paper be used in a meta-analysis? Make the reach of your research broader and longer lasting. Methods Ecol. Evol. 8, 777-784 (2017).

10. Holcombe, A. Farewell authors, hello contributors. Nature 571, 147-147 (2019). 
11. Elliott, J. H. et al. Living Systematic Reviews: An Emerging Opportunity to Narrow the Evidence-Practice Gap. PLOS Med. 11, e1001603 (2014).

12. Nakagawa, S. et al. Research Weaving: Visualizing the Future of Research Synthesis. Trends Ecol. Evol. 34, 224-238 (2019).

13. Westgate, M. J. et al. Software support for environmental evidence synthesis. Nat. Ecol. Evol. 2, 588-590 (2018).

14. Haddaway, N. R. \& Crowe, S. Experiences and lessons in stakeholder engagement in environmental evidence synthesis: a truly special series. Environ. Evid. 7, 11 (2018).

15. Haddaway, N. R. Open Synthesis: on the need for evidence synthesis to embrace Open Science. Environ. Evid. 7, 26 (2018).

16. Wilkinson, M. D. et al. The FAIR Guiding Principles for scientific data management and stewardship. Sci. Data 3, 160018 (2016).

17. Longo, D. L. \& Drazen, J. M. Data Sharing. N. Engl. J. Med. 374, 276-277 (2016).

18. West, J. D., Jacquet, J., King, M. M., Correll, S. J. \& Bergstrom, C. T. The Role of Gender in Scholarly Authorship. PLOS ONE 8, e66212 (2013).

19. Grogan, K. E. How the entire scientific community can confront gender bias in the workplace. Nat. Ecol. Evol. 3, 3-6 (2019).

20. Freeman, R. B. \& Huang, W. Collaboration: Strength in diversity. Nature 513, 305 (2014).

21. Begley, C. G. \& Ellis, L. M. Drug development: Raise standards for preclinical cancer research. Nature 483, 531-533 (2012).

22. Sholler, D., Ram, K., Boettiger, C. \& Katz, D. S. Enforcing public data archiving policies in academic publishing: A study of ecology journals. Big Data Soc. 6, 2053951719836258 (2019).

23. Culina, A., Crowther, T. W., Ramakers, J. J. C., Gienapp, P. \& Visser, M. E. How to do meta-analysis of open datasets. Nat. Ecol. Evol. 2, 1053-1056 (2018).

24. Kidwell, M. C. et al. Badges to Acknowledge Open Practices: A Simple, Low-Cost, Effective 
Method for Increasing Transparency. PLOS Biol. 14, e1002456 (2016).

25. Vazire, S. Implications of the Credibility Revolution for Productivity, Creativity, and Progress. Perspect. Psychol. Sci. 13, 411-417 (2018). 


\section{Consortium Authorship}

Evidence Synthesis Hackathon 2019 Participants

Matthew Carter ${ }^{3}$

Owen Forbes ${ }^{17}$

Luis Furuya-Kanamori ${ }^{17}$

Charles T. Gray ${ }^{18}$

W. Kyle Hamilton 19

Fonti Kar ${ }^{1}$

Emily Kothe 20

Joey Kwong 21

Luke A. McGuinness 22

Paige Newman ${ }^{2}$

Mandlenkosi Ngwenya ${ }^{23}$

Christopher Penkin ${ }^{24}$

Daniel Perez ${ }^{25}$

Michael Schermann ${ }^{26}$

Alistair Senior 27

Juan Vásquez 25

Wolfgang Viechtbauer ${ }^{28}$

Thomas White 27

Mitchell Whitelaw ${ }^{29}$

\section{Affiliations}

${ }^{1}$ Evolution \& Ecology Research Centre, School of Biological, Earth and Environmental

Sciences, University of New South Wales, Sydney, NSW, Australia

${ }^{2}$ Centre for Health Informatics, Macquarie University, Sydney, NSW, Australia

${ }^{3}$ Centre for Research in Evidence-Based Practice, Bond University, Gold Coast, QLD, Australia

${ }^{4}$ Department of Ecology \& Evolutionary Biology, University of Connecticut, Connecticut, USA

${ }^{5}$ Department of Evolutionary Biology, Bielefeld University, Germany

${ }^{6}$ Division of Ecology and Evolution, Research School of Biology, The Australian National University, Canberra, ACT, Australia

${ }^{7}$ Fenner School of Environment \& Society, The Australian National University, Canberra, ACT, Australia

${ }^{8}$ Australian Academy of Science, Canberra, ACT, Australia

${ }^{9}$ College of Medicine and Health, University of Exeter, UK

${ }^{10}$ Department of Psychological Sciences, University of Connecticut, Connecticut, USA

${ }^{11}$ Centre for Health Policy, School of Public Health, University of Witwatersrand, South Africa

${ }^{12}$ School of Mathematical Sciences, Queensland University of Technology, Brisbane, QLD,

Australia

${ }^{13}$ School of Public Health and Preventive Medicine, Monash University, Melbourne, VIC, Australia

${ }^{14}$ Campbell Collaboration

${ }^{15}$ Stockholm Environment Institute, Linnégatan 87D, Stockholm, Sweden

${ }^{16}$ Africa Centre for Evidence, University of Johannesburg, Johannesburg, South Africa

Below this point affiliations only appear in the supplementary material 
${ }^{17}$ National Centre for Epidemiology and Population Health, Research School of Population Health, The Australian National University, Canberra, ACT, Australia

${ }^{18}$ Department of Mathematics and Statistics, School of Engineering and Mathematical Sciences,

La Trobe University

${ }^{19}$ Psychological Sciences, University of California, Merced, California, USA

${ }^{20}$ School of Psychology, Deakin University, Melbourne, VIC, Australia

${ }^{21}$ United Nations Population Funds Asia and the Pacific Regional Office, Hong Kong

${ }^{22}$ Department of Population Health Sciences, Bristol Medical School, Bristol, United Kingdom

${ }^{23}$ South African Department of Planning, Monitoring, and Evaluation, South Africa

${ }^{24}$ Digital Solution Foundry, Johannesburg, South Africa

${ }^{25}$ Epistemonikos Foundation, Santiago, Chile

${ }^{26}$ Department of Information Systems \& Analytics, Santa Clara University, Santa Clara, CA, USA

${ }^{27}$ School of Life and Environmental Sciences, The University of Sydney, Sydney, NSW, Australia

${ }^{28}$ Department of Psychiatry and Neuropsychology, School for Mental Health and Neuroscience, Maastricht University, The Netherlands

${ }^{29}$ School of Art and Design, The Australian National University, Canberra, ACT, Australia

\section{Acknowledgements}

Evidence Synthesis Hackathon 2019 was supported by the Faculty of Science at UNSW Sydney, Fenner School of Environment \& Society at the Australian National University, Stockholm Environment Institute, and University of Johannesburg. AST was partially funded by the Deutsche Forschungsgemeinschaft (DFG, German Research Foundation) - Projektnummer 316099922 - TRR 212.

\section{Competing interests}

The authors declare no competing interests. 